Las mujeres extranjeras en las nuevas ruralidades

Foreign women in new ruralities

Montserrat Soronellas

Profesora Titular. Departamento de Antropología, Filosofía y Trabajo Social. Universitat Rovira i Virgili. Tarragona (España)

Parianontserratsoronellas@urv.cat

Yolanda Bodoque

Profesora Lectora. Departamento de Antropología, Filosofía y Trabajo Social. Universitat Rovira i Virgili. Tarragona (España) yolanda.bodoque@urv.cat

Ramona Torrens

Profesora Colaboradora. Departamento de Antropología, Filosofía y Trabajo Social. Universitat Rovira i Virgili. Tarragona (España)

ramona.to

NUEVAS RURALIDADES

WOAE

Este artículo analiza impacto de la llegada de población inmigrada en áreas rurales catalanas desde una perspectiva de Este articulo analiza impacto de la llegada de poblacion intigrada en areas rurals catalanas desde una perspectiva de genero. En concreto hemos observado los itinerarios migratorios femeninos a municipios con problemas para garantizar la planteando que estas mujeres contribuyen de forma singular a su desarrollo al suplir parte del capital humano y laboral perdido en el proceso de declive característico de las zonas rurales durante el siglo XX. Hemos observado las tendencias demográficas de la inmigración al medio rural y su caracterización, los itinerarios migratorios, expectativas y la singularidad de su incorporación a un medio tan específico social, económica y culturalmente. A diferencia de la migración masculina que en estas zonas suele ocuparse en el sector primario, las mujeres trabajan prioritariamente en el sector servicios (hostelería turismo), en el sector agrotransformador y, especialmente, en trabajos relacionados con la esfera reproductiva, el cuidado y atención a la población envejecida que, a causa del exodo rural, no suele tener un contexto familar (ni instivational) cercano resuelva sus necesidades de atencion. Entendemos que sociales esenciales.

ABSTRACT

This article analyzes the impact of the arrival of immigrant population in the Catalan rural areas from a gender perspective. We have particularly observed the female migratory routes to municipalities with problems in ensuring the reproduction of their communities (depopulation, masculinization, aging, singleness, economic marginality) suggesting that these women contribute uniquely to its development and the revival of part of human capital and labor lost in the decline process that has characterizes the rural areas throughout the twentieth century. We noted the demographic trends of immigration to rural areas and their economically and cuturally unlike mace migration in these ars which is usually concerned in the primary sector, socially, work primarily in the service sector (hotels and tourism), in the agro processors and especially in work related to the reproductive sphere, care and attention to the aging population who because of rural exodus, do not usually have either a family or institutional context that meets their needs of close attention. We understand that the foreign women help to re feminize these communities and become essential economic and social agents.

PALABRAS CLAVE

migraciones internacionales | migraciones femeninas | sociedad rual | atención y cuidado | estudios de género

international migration | female migration | rural society | attention and care | gender studies

Este artículo (1) analiza las condiciones de incorporación de mujeres extranjeras inmigradas a las zonas rurales catalanas. Nos ha interesado la observación de las migraciones femeninas a unos municipios que tienen desde hace décadas problemas para garantizar la reproducción de sus comunidades (despoblación, masculinización, envejecimiento, soltería, marginalidad económica). Como hipótesis de partida planteamos que estas mujeres debían de contribuir de forma singular al desarrollo y a la reproducción de las comunidades, puesto que suplen parte del capital humano y laboral que la comunidad ha perdido en el proceso de declive demográfico característico de las zonas rurales durante el siglo XX. A diferencia de la migración masculina que en las zonas rurales tiende a ocuparse en el 
sector primario, las mujeres trabajan prioritariamente en el sector servicios (hostelería y turismo), también en el sector agrotransformador $y$, muy especialmente, en trabajos relacionados con la esfera reproductiva: el cuidado y atención a la población envejecida de los pueblos que, a causa del éxodo rura (la emigración de los hijos e hijas) no suele tener un contexto familiar, ni institucional, cercano que resuelva sus necesidades de atención. Entendemos que las mujeres extranjeras llegadas a los pueblos contribuyen a refeminizar las comunidades y devienen agentes económicos y sociales esenciales en cualquiera de las modalidades de desarrollo rural con las que nos hemos encontrado a lo largo de la investigación, las cuales exploramos en el apartado 3 de este artículo.

Para ello se realizó un análisis demográfico de la población rural catalana (con datos procedentes de Padrón de habitantes a 1/01/2010) así como una tipología de municipios rurales catalanes de menos de 2.000 habitantes, que nos permitió definir las características a partir de las cuales escogimos los doce pueblos donde realizamos el trabajo de campo. Los criterios de selección fueron: tener más de un $10 \%$ de poblón exocial y económico del municipio. Se realizaron 78 entrevistas a mujeres extranjeras, a contratadores y agentes locales (lécnicos de inmigración, alcaldes, concejales y maestros de escuela y otras personas con quienes mantuvimos conversaciones informales) con las que hemos intentado cubrir todas las situaciones y perfiles posibles hasta que llegamos a la saturación informativa teniendo en cuenta los objetivos que nos habíamos propuesto. La observación participante se llevó a cabo tanto en espacios públicos de los municipios (calles, plazas, bares, comercios...) y privados (domicilios particulares) y se han recogido datos mediante la elaboración de diagramas de parentesco y representaciones gráficas de redes.

Entre los objetivos de nuestra investigación contemplamos la caracterización de la población extranjera que llega a los pequeños municipios (2), sus itinerarios migratorios, sus expectativas y, muy especialmente, la singularidad de su proceso de incorporación a un medio con especificidades sociales, económicas y culturales que marcan diferencias respecto a los entornos urbanos que, por ser grandes receptores de población inmigrada, han sido mucho más estudiados. En el análisis de las formas de incorprán a los puón in en nuestra soción en nuestra sociedad: que la incorporación ventajosa para la población inmigrada que la incorporación a la gran ciudad.

\section{La nueva ruralidad y las migraciones}

La población extranjera emigrada que en las últimas décadas ha llegado a las zonas rurales ha significado un revulsivo social, económico y demográfico para nos pueblos que llevaban décadas inmersos en un proceso de pérdida constante de población, de declive económico y de empobrecimiento del tejido y de las dinámicas sociales (García Sanz 2006). La situación de crisis de las zonas rurales ha de ser explicada atendiendo a un complejo de factores: la pérdida de competitividad económica de las rentas agrarias; el descrédito de la cultura y de las formas de vida rural y campesina frente a los modelos urbanos; las condiciones de aislamiento de algunas zonas rurales; las limitaciones del mercado de trabajo y la falta de oportunidades, entre otras (Etxezarreta y Viladomiu 1997, Soronellas 2006). No obstante, a inicios del siglo XXI, la población rural se está estabilizando e incluso, en algunas zonas, se recupera tímidamente gracias a la combinación de algunos factores que favorecen la fijación de población en el fentes de desarrollo econos industria agroalimentaria y artesana) y la mejora de las comunicaciones y de las condiciones de acceso de los habitantes de las zonas rurales a los servicios básicos. La llegada de población inmigrada extranjera atraída tanto por la demanda de mano de obra del sector agrícola, como por las nuevas ocupaciones del sector terciario rural, contribuye también a la consecución de saldos demográficos positivos por parte de muchos pequeños municipios, más acostumbrados a perder población que a recibir nuevos vecinos. Estamos, pues, ante un proceso de repoblación de algunas zonas rurales (Esparcia 2002, García Sanz 2003, García Coll y Sánchez 2005, Camarero 2009).

Interesa poner de relieve, al respecto de nuestro objeto de estudio, que esta repoblación supone también la emergencia de un cierto proceso de nueva feminización de unos espacios rurales que perdieron en primer lugar a muchas de sus mujeres, las cuales huían del campo en busca de recursos económicos y, también, de un nuevo rol social que las dejase más espacio de participación y de decisión. La llegada de mujeres al medio rural compensa la masculinización endémica de muchos pequeños municipios, no sólo 
Las migraciones internas campo-ciudad también han modificado la demografía de los municipios no urbanos. El llamado "éxodo urbano" ha movido las poblaciones desde las ciudades hacia los pueblos situados en las áreas de influencia de éstas (Morén Alegret y Solana 2006). No obstante, la relación ciudad-campo no se limita exclusivamente a las migraciones internas y a la existencia de población flotante, también hemos de tener en cuenta que el desarrollo de la sociedad industrial y capitalista ha creado nuevas necesidades de consumo fundamentadas en el ocio y en la oferta turística. Esta diversificación económica alimentada por los programas de desarrollo rural financiados por programas específicos (Leader y Proder, por ejemplo) ha creado el contexto económico ideal para la fijación de la población local en los pueblos, pero también para ejercer como polos de atracción de población nueva: contexto de oportunidad laboral y de vida.

\subsection{Migraciones de mujeres: motivaciones, trayectorias y cadenas migratorias}

El papel de las mujeres en los flujos migratorios internacionales se remonta a finales de la década de los setenta y siguió la misma tendencia que en el resto de países receptores de población migrada: primero fue invisibilizado o tratado de manera secundaria en los estudios clásicos sobre migraciones, y no es hasta finales de los noventa que adquiere un protagonismo relevante. La invisibilidad era producto de pensar las migraciones entre naciones atendiendo únicamente a los factores de expulsión y atracción de mano de obra, fruto de la causalidad económica y protagonizadas por hombres que realizaban una migración asociada a su papel de breadwinners, mientras que las mujeres los seguían, reagrupadas y dependientes económicamente. Los estudios más actuales otorgan importancia a los procesos estructurales (macro), pero también a la decisión personal y familiar (micro). Visibilizan las migraciones pensadas y protagonizadas por mujeres; superan el nacionalismo metodológico (Suárez 2008); hacen irrumpir con fuerza la mirada transnacional que atiende a los flujos y a las relaciones de los migrantes con sus lugares de origen. Las investigaciones recientes toman en consideración la causalidad diversa de mediambientales (King 2002) e incluso por amor (Roca y otros 2008).

Las condiciones estructurales para decidir una migración puede que sean genéricamente económicas. De hecho los colectivos migratorios con mayor presencia en Cataluña y España (procedentes de Latinoamérica, Europa del Este y norte de África) comparten, cada uno con sus particularidades, situaciones socioeconómicas similares en sus países de origen que contribuyeron a la expulsión de población: dificultades económicas, desestabilización política e inseguridad ciudadana, crisis políticas y económicas, posición periférica en la esfera global, analfabetismo, etc. Las mujeres evidencian otros estímulos estructurales a su movilidad: un marcado sistema patriarcal; la división internacional del trabajo en función del género que fomenta o excluye su ocupación en determinados sectores; la situación de subordinación en el ámbito doméstico; o el lugar que ocupan respecto a los hombres de su país, entre otros (Gregorio 1998). Hablar solamente de migración económica simplifica la realidad ya que no se toma en consideración la migración como proyecto personal y como contexto de oportunidad también social.

Enfocar la atención en las mujeres ha puesto de manifiesto varios trasfondos de la migración: el familiar y el personal. El grupo familiar puede ser un estímulo a la emigración cuando resulta ser un mecanismo de ( compromiso del sostenimiento del grupo doméstico (Gregorio 1998). Entonces dejan a su familia en e pais de origen, hijos y conyuge especialmente, y ejercen to que se ha calificado como maternidad transnacional (Bryceson y Vuorela 2001, Ramirez 2002, Parrenas 2005, Parella 2007, Sanz 2007 Pedone 2003), es decir, desde la distancia. Son mujeres que llegan mirando atrás, hacia la parte de familia que quedó en la otra parte de su nuevo campo social. Pero tampoco hay que olvidar lo personal, 0 que Agustín (2003) ha denominado los "estados del alma", el deseo de cambiar, mejorar las condiciones de vida, huir de estados de frustración y angustia; otros hablan de la existencia de una cultura migratoria arraigada en el entorno familiar, local o nacional que favorece la movilidad e impulsa la conquista de derechos y privilegios perdidos e irrecuperables a corto plazo en el propio entorno (Arellano 2006): son las migrantes que llegan mirando hacia adelante, hacia la sociedad donde piensan forjar su destino. Pero incluso en estos casos el trasfondo familiar de la migración es importante porque de cualquier manera las migrantes no dejan de ser las representantes de sus familias en el extranjero, y su grupo familiar de manera más o menos intensa está presente en la elaboración de su trayectoria migratoria.

De hecho, sus proyectos están respaldados por cadenas y redes migratorias (3), una categoría analítica 

migran personas, familias y colectivos nacionales también lo hacen ideas y posiciones simbólicas de sus miembros donde podemos encontrar tanto solidaridad y cooperación como relaciones de poder, jerarquía y dominio de la sociedad patriarcal. Quien decide migrar puede seguir una cadena de parientes, amigos o paisanos que ya lo han hecho antes que ellos, o bien iniciar una nueva. Respecto a la composición de estos entramados sociales (4), el Colectivo IOÉ (1996) afirma que en las redes que facilitan la migración el género actúa de diversas maneras en función de si son de predominio masculino (las mujeres migran a instancias del marido o aprovechando la presencia de hombres de la familia en el país de destino): de predominio femenino (tanto en origen como en destino formadas por mujeres que facilitan información,

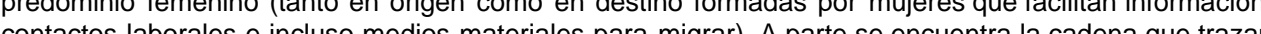
contactos laborales e incluso medios materiales para migrar). A parte se encuentra la cadena que trazan los empleadores que alimenta la segmentación laboral en función del género al colocar mujeres como

\subsection{La crisis del cuidado}

En las últimas décadas, los Regímenes de Bienestar Mediterráneos han vivido unos procesos de cambio que tienen fuertes repercusiones sobre las formas familistas tradicionales de canalizar el bienestar y que han dado lugar a lo que algunos especialistas llaman crisis of care (Bettio et al. 2004) o care deficit (Hochschild 2001, Degiuli 2007). Los cambios demográficos, culturales, sociales y económicos de las últimas décadas, producidos principalmente por la incorporación masiva de la mujer en el mercado de trabajo formal y por el envejecimiento de la población, suponen la crisis de la familia patriarcal y de la forma de gestionar el cuidado (Solé 2008). Se establece un vínculo entre la inmigración y el envejecimiento que emerge de la necesidad de reorganizar el trabajo reproductivo a nivel macro -entre la familia, el Estado y el mercado-, y a nivel micro -entre géneros y generaciones dentro del núcleo familiar(Letablier 2007) (5). Esta nueva división del trabajo entre familia, mercado y Estado refuerza la lógica del mercado y potencia su papel en relación a este sector (Martínez Virto 2010, Martínez Buján 2010): una mercado y por cla su papel en relación a este sector (Martízz Vin 2010, Manthez Buján 2010): una mercantilización progresiva de la atención a la dependencia; las familias externalizan la atención a sus familiares mayores hacia el servicio doméstico, lo que supone la creación y mantenimiento de un nicho laboral precario e inestable caracterizado por una regulación obsoleta de los trabajos de atención a las personas. Las trabajadoras inmigrantes asumen gradualmente, a través de un mercado que opera a escala global, una parte de los trabajos de cuidado remunerados, configurando un modelo migratorio capaz de satisfacer las insuficiencias de servicios para este sector.

La necesidad de mano de obra específica para realizar los trabajos reproductivos incide como mecanismo selector y moldeador de los flujos migratorios, reflejando procesos de dimensiones globales transnacionales como la mundialización de los trabajos reproductivos de las familias -lo que se refiere como las cadenas mundiales del afecto y el cuidado (Hochschild 2001) o las cadenas globales del cuidado, y la feminización de los circuitos globales de supervivencia (Sassen 2003) que refleja cómo las mujeres se remplazan en los trabajos de cuidado y atención a las personas. En el escenario actual la gestión del cuidado pasa a ser una cuestión que traspasa los límites nacionales y poco a poco se inserta en un proceso de transnacionalización del servicio doméstico femenino o de un sistema de transmisión global del cuidado (Parella 2003, Oso 1998). Desde esta perspectiva de la internacionalización del trabajo reproductivo es posible romper con el paradigma productivo más ortodoxo basado en la racionalidad económica y aprender la importancia de la economía del cuidado para el funcionamiento de racionalidad económica y aprender la importancia de la economía del cuidado para el funcionamiento de
la sociedad (Benería 2006). En este sentido, las mujeres inmigrantes son parte de la circulación de recursos, de capital y de trabajo (Castelló 2008).

\section{Las migraciones internacionales en las zonas rurales catalanas}

En términos generales, la población (6) de las zonas rurales ha ido creciendo de manera similar al tota de Cataluña (7), algo muy significativo si se tiene en cuenta que en los últimos decenios la pérdida de población parecía imparable. Parte de este crecimiento se debe a los desplazamientos ciudad-campo (Morén-Alegret y Solana 2006), aunque el aumento por la incorporación de población extranjera ha sido el más destacable en la última década en los pequeños municipios: un $2.5 \%$ de residentes nacidos en e extranjero en 2000 , frente al $10.7 \%$ del año 2010 , un porcentaje inferior al del total de Cataluña (16.3\%) pero muy significativo. El incremento demográfico afecta al $75 \%$ de los 600 municipios que en Cataluña tienen men 2.000 ina en Cataña tienen 
La comparación de las pirámides de la población residente en los municipios rurales catalanes en 2000 y 2010, evidencia el crecimiento mencionado y el rejuvenecimiento de la población rural por el incremento del grupo de jóvenes y un cierto retroceso de los grupos de población de más edad (8). Es notorio también el incremento de la natalidad y la existencia de población infantil llegada con los procesos de reagrupación familiar de los inmigrantes. Debemos destacar también que, a pesar de la incorporación de mujeres extranjeras, la tendencia a la masculinización de las zonas rurales no se corrige, básicamente como consecuencia de la incidencia de la llegada a los pueblos de un mayor número de hombres extranjeros atraídos por la oferta de trabajo agrícola.

En cuanto a los países de origen de la población extranjera residente en los municipios rurales catalanes, los más representados son rumanos (25.5\%) y marroquíes (24.5\%), seguidos de latinoamericanos (19\%) y de personas originarias de otros países de la UE (17\%). El colectivo rumano, que representa tan sólo el y\% del total de polón in $9 \%$ del total de población inmigrada extranjera en Cataluna, tiene más tendencia a inmigrar a las zonas rurales $y$, es también el colectivo que se halla más distribuido por todo el territorio de la Comunidad Autónoma. En cuanto a la distribución por sexos, el $45 \%$ de la población inmigrada residente en los pequeños municipios son mujeres. La inmigración latinoamericana, con un patrón de inmigración más urbano, y más especializada en el sector servicios, especialmente en los servicios personales y de ámbito doméstico, está más feminizada (alrededor del 60\% son mujeres). La población marroqui inmigrada a los pequeños municipios muestra, a pesar de las reagrupaciones familiares, una tendencia a la masculinización que es mucho más evidente en el colectivo subsahariano, donde el $90 \%$ de los inmigrantes son de sexo masculino.

\section{La inmigración de mujeres extranjeras a las nuevas ruralidades}

En términos generales, las mujeres ha ocupado históricamente una posición subordinada en las sociedades agrarias, las cuales han funcionado a partir de estructuras patriarcales invisibilizando la labor desarrollada por éstas en las unidades de producción familiar y/o como trabajadora asalariada del campo. Su posición marginal, aunque esencial, explica que hayan sido precisamente ellas las primeras en abandonar los pueblos, ya desde finales del siglo XIX. La emigración campo-ciudad ha despoblado las zonas rurales y ha dificultado la reproducción de las comunidades, una realidad acentuada en zonas de montaña, por la mayor incidencia de la emigración femenina que ha dejado a los hombres solteros y sin mercado matrimonial (Bourdieu 2004, Bodoque 2009).

La intensificación productiva propia de la agricultura del siglo XX, ha transformado también el modelo de participación de las mujeres en las labores del campo y su implicación en las explotaciones agrarias. Las que permanecen en los pueblos han redefinido su participación en la economía agraria y en la comunidad. Desde una profunda implicación en el trabajo agrario de la época preindustrial a un distanciamiento de las labores del campo y una mayor especialización en el trabajo reproductivodoméstico de la época industrial, hasta llegar a hoy donde su posición pendula entre la implicación económica y laboral en el sector agrario o en algunos de los sectores económicos propios de la nueva ruralidad (servicios y agrotransformación), y sus responsabilidades en las tareas reproductivas enmarcadas en su rol de género más tradicional. Las políticas de desarrollo rural impulsadas por la administración persiguen implicar a las mujeres e impulsarlas en su papel de emprendedoras de iniciativas socioeconómicas y laborales y en su rol de agentes del desarrollo local.

La administración persigue fomentar la emprendeduría de las mujeres de las zonas rurales pero los pueblos adolecen de falta de mujeres, incluso cuando los proyectos de desarrollo local consiguen salir pueblante. Las economías rurales, la despoblación y el envejecimiento, han creado un contexto de oportunidad para la población inmigrada que encuentra en los pueblos empleos precarios y mal oportunidad para la población inmigrada que encuentra en los pueblos empleos precarios y mal
remunerados que resultan poco atractivos a la población local: las labores agrícolas o agroindustriales remunerados que resultan poco atractivos a la población local: las labores agrícolas o agroindustriales
(Pedreño y Riquelme 2007, Reigada 2007, Gualda 2004, Arellano 2006), la hostelería o la atención a la dependencia, son los sectores de mayor demanda de mano de obra femenina. A la precariedad de las condiciones laborales, añadimos la dificultad de vivir y trabajar en zonas alejadas y frecuentemente ma comunicadas. A pesar de todo, las mujeres llegan a las zonas rurales y permanecen en ellas. ¿Que ruralidades encuentran al llegar?, ¿cómo son los pueblos donde fijan su nueva vida? Hemos hallado contextos rurales distintos en función de cómo se establece la relación entre el modelo de desarrollo local y la presencia de población inmigrada, especialmente de mujeres y hemos identificado cuatro categorías de ruralidad, cada una de las cuales atrae e incorpora a mujeres procedentes de países extranjeros. 

pueblos agrícolas cuyos habitantes han renovado, tecnificado, especializado y/o reconvertido las empresas agrarias. En esta tipología, podemos situar dos tendencias distintas: los pueblos que han especializado sus cultivos con técnicas de explotación intensivas y aquellos donde se han desarrollado proyectos agrícolas nuevos que han perseguido el objetivo de diferenciar las producciones agrarias buscando conseguir cotas de calidad (gastronómica, medioambiental o de salud) con las que aumentar e valor de mercado de los productos alimentarios. Entre los que han intensificado los cultivos, seleccionamos el caso de Benissanet, en la Ribera d'Ebre (1.263 hab.), cuya agricultura está dedicada a la producción frutícola intensiva, lo que le ha permitido sortear la despoblación y atraer a población inmigrada (9). Hasta Benissanet llegan mujeres contratadas en origen en Eslovaquia y Lituania para inmigrada (9). Hasta Benissanet llegan mujeres contratadas en origen en Eslovaquia y Lituania para trabajar temporalmente en los almacenes de preparación y envasado de la fruta de la cooperativa local Han llegado también por su propia cuenta, mujeres rumanas que trabajan en los servicios (hostelería servicio doméstico, cuidado de personas mayores) y marroquíes que están ocupadas en las tareas de recogida de la fruta. En cuanto a las zonas agrícolas revalorizadas a partir de la consecución de producciones de calidad, tomamos el ejemplo del municipio de la Morera de Montsant (159 hab.) (10), un pueblo situado en el Priorat, una comarca que en los últimos 20 años ha resurgido con la implantación en el territorio de empresas vinícolas que han sido el factor de dinamización de la comunidad (11) y de aportación de población joven inmigrada para trabajar en los viñedos, en las bodegas y en alguna actividad de hostelería que ha surgido en la zona al abrigo del enoturismo.

Otra ruralidad es la de aquellos municipios que mantienen la actividad agrícola con el apoyo de un sector industrial que contribuye a la diversificación y al sostenimiento de la economía local. Son pueblos bien ubicados respecto a vías de comunicación y a zonas urbanas e industriales. En Vila-rodona (1.298 habitantes), en la comarca de l'Alt Camp (Tarragona) en la última década del siglo XX, empezaron a llegar hombres marroquíes para trabajar en las labores del campo (viñedos y algunos frutales) que, con los años, reagruparon a sus familias y que han fijado su residencia en la localidad (161 marroquíes los años, reagruparon a sus familias y que han fijado su residencia en la localidad (161 marroquies
residentes). La gran mayoría de mujeres marroquíes no se han incorporado al mercado de trabajo, residentes). La gran mayoría de mujeres marroquíes no se han incorporado al mercado de trabajo,
algunas trabajan ocasional y estacionalmente en la recogida de la fruta. El despliegue de la zona algunas trabajan ocasional y estacionalmente en la recogida de la fruta. El despliegue de la zona
industrial ha ocupado a la población local, otrora dedicada a la actividad agraria, mientras la población extranjera llegada a Vila-rodona sigue teniendo su nicho laboral en la agricultura. A partir de 2005, llegan mujeres rumanas y latinoamericanas que se colocan en la hostelería y en el servicio doméstico. Actualmente el $20 \%$ de la población de Vila-rodona es extranjera.

Una tercera tipología de zona rural es aquella en la que prácticamente ha desaparecido el sector primario y donde se ha desarrollado un sector terciario especialmente dedicado al turismo. En esta situación encontramos las zonas más alejadas de las áreas urbanas y con mayores valores de interés turístico: paisajes, clima y patrimonio cultural, fundamentalmente. En este grupo destacamos los municipios de Les, en el Pirineo de Lida; Prades, en Tarragona; o Organyà en Lleida. Todos ellos son un buen ejemplo de ) tiene un $28 \%$ de ponjer femenina. Les ( $1.011 \mathrm{hab}$.) tien un $28 \%$ de población extranjera, 282 personas que empezaran a llegar en 2004. Las pimeras fueron mujeres (bolvianas), reclutadas a traves de cadenas migratorias muy activas, que encontraron trabajo en un sector comercial muy activo dado el carácter fronterizo de municipio. Los comercios de Les atraen población francesa que acude en busca de precios más económicos (en el tabaco y el alcohol, fundamentalmente). En el caso de Prades estamos ante un pueblo de montaña con atractivos interesantes, paisajísticos y patrimoniales, que le han dado prestigio como zona de ocio y descanso próxima, aunque suficientemente alejada, de la zona metropolitana industrial de Tarragona. Tanto es así que en los últimos 30 años, el pueblo ha crecido gracias a la construcción de segundas residencias, las cuales han propiciado el surgimiento de numerosos establecimientos comerciales, de restauración y de hostelería que satisfacen las necesidades de servicios de visitantes, turistas y residentes ocasionales. El empresariado del sector terciario de Prades es la misma población local, pero los trabajadores asalariados que sostienen los establecimientos son, es la misma pobcion local, pero los trabajadores asal casi mi 100\%, población ex laborales. En Prades viven 665 habitantes, de distintos, aunque más de la mitad son rumanos.

Por último, algunos pueblos responden al modelo rural propio del último cuarto del siglo XX: despoblados y envejecidos, donde persiste cierta actividad agrícola tradicional y marginalizada y sin una alternativa de desarrollo rural capaz de frenar el proceso. Ni la administración local, ni el tejido social y económico han liderado proyectos nuevos. Tres de los municipios analizados responden a esta tendencia (12). Por 
ejemplo Prat de Comte (201 hab.), en la comarca de la Terra Alta (Tarragona), cuya actividad económica principal sigue siendo la agricultura de secano, que cuenta con una población envejecida y donde no ha surgido ninguna iniciativa, pública o privada, que haya permitido desarrollar alguna actividad terciaria 0 artesano-industrial. A pesar del pobre tejido social y económico, el pueblo ha recibido población extranjera en los últimos años: hombres, ocupados en labores del campo (pakistaníes y marroquíes, principalmente) y mujeres que trabajan en la atención a la dependencia de las personas mayores, uno de los principales nichos laborales para las mujeres extranjeras en los pequeños municipios de estas características. Habituada a perder población desde principios de siglo XX, Prat de Comte ha recuperado población en los últimos 10 años gracias a la llegada de las 24 personas extranjeras que actualmente viven en la localidad, pero estas incorporaciones no resuelven la reproducción de la comunidad a largo plazo.

En síntesis, las diversas ruralidades de los municipios de menos de 2.000 habitantes de Cataluña han sido receptoras de po sido remanda fundam masculina, pero los procesos de terciarización de las economías rurales, principalmente vinculados a turismo, han favorecido la llegada a los pueblos de mujeres que trabajan en el comercio y la hostelería. En todas las formas de ruralidad, hemos encontrado mujeres ocupadas en los servicios domésticos y personales, especialmente en la atención a la población envejecida que, a consecuencia de la despoblación secular, caracteriza la demografía de las zonas rurales.

\section{El medio rural como contexto de oportunidad para mujeres inmigradas}

En el análisis las trayectorias de la migración femenina hacia las áreas rurales catalanas hemos detectado una serie de singularidades relacionadas con las condiciones que ofrecen a las mujeres los contextos de llegada. Entre estas singularidades destacamos cómo los pueblos visibilizan las cadenas migratorias, hacen emerger el territorio más allá de la comunidad, determinan la movilidad laboral y territorial de las mujeres; y también sus condiciones de incorporación al pueblo.

\subsection{Las cadenas visibles}

En la primera fase del trabajo de campo, la de la selección de los pueblos, descubrimos la presencia de pequeños colectivos nacionales distintos entre localidades relativamente cercanas. Sin un orden aparente, observamos que en cada pueblo predomina demográficamente un grupo nacional, el cual puede ser muy distinto del que predomina en el municipio vecino. Algunos colectivos de origen los encontramos en la mayor parte de municipios (el rumano, especialmente) y nos sorprendió que otros fueran de procedencias poco convencionales en el mapa migratorio catalán: portugueses, moldavos, rusos, búlgaros, checos, paraguayos, hondureños, ucranianos o salvadoreños. La importancia numérica rusos, búlgaros, checos, paraguayos, hondureños, ucranianos o salvadoreños. La importancia numérica de estos colectivos es poco significativa, pero en el contexto de la pequeña comunidad adquieren
visibilidad y, más importante, visibilizan las cadenas, frecuentemente familiares, que han vehiculado la migración de cada uno de los miembros del colectivo. Al acercamos a las trayectorias migratorias de las mujeres entendemos la activación de cadenas y redes personales, laborales y/o familiares.

Las cadenas migratorias analizadas son diversas: amplias y complejas o cortas y simples; algunas con muchos eslabones, otras con muy pocos; algunas muy femeninas, otras no tanto; y todas inacabadas mientras haya en origen alguna persona con deseo migratorio. Una cadena amplia y compleja acostumbra a ser familiar, arrastra grupos domésticos enteros hacia una misma localidad, dejando en evidencia proyectos migratorios netamente familiares (Suárez y Crespo 2007). Las familias de Bernarda (Portugal, Vila-rodona), Tatiana (Ucrania, Freginals) o Pilar (Bolivia, Les) entre otros, ejemplifican la llegada sucesiva de familiares que ha reforzado la cadena y que ha convertido a la familia en una gran red acogedora y a su colectivo nacional en el más significativo del municipio. En el pueblo se vive y trabaja, pero la comunidad relacional acostumbra a ser la propia red familiar:

"Aquí vino primero mi cuñada que vino por su hermano que era tractorista, hace ya 11 años después mi hermano y todos vinieron al pueblo (...) Mi hijo y mi sobrino vinieron también (...) de nuestra población luego vino mi cuñada y la prima hermana y casi toda la familia, consuegros, suegros, primos, sobrinos (...) nos vinimos por saber que hay alguien que te espera, que te da la comida (...) Mi hijo vino en junio de 2001, se casó después allí, tiene dos hijas que están allá después vino la sobrina soltera se casó aquí con un lituano tiene dos hijos nacidos aquí, después vine yo y mi marido en 2001, en 2006 mi hijo mayor se casó allí y vino con la mujer, tienen el petit freginalenc y el hijo menor se casó allá, vino casado con un hijo" (Tatiana, Ucrania, Freginals). 

capital social, puesto que su experiencia local la convierte en una valiosa fuente de información para acceder a trabajos o para facilitar la movilidad laboral de los miembros de la familia.

"Yo trabajaba en el supermercado y mi marido cuando llegó ya no trabajó en el supermercado sino en otro negocio de mi jefe y ahí está ahora. Luego ya empezaron a venir mis cuñados, que empezaron a trabajar en el Claravall porque me dijeron que necesitaban gente y no había muchos extranjeros y allá te decía si podía venir... entonces vino una de mis primas, luego mi otra prima, con su marido, luego mi hermana con su marido (...) me pedían consejo y yo les ayudaba todo lo que podía, con los trámites, les decía que tienen que hacer este paso y me llamaban 'pero ¿cómo tengo que hacer los papeles?' pues primero te tienen que dar la oferta, más o menos me lo sé ya" (Pilar, Bolivia, Les).

Otro tipo de redes son las formadas por diversos núcleos familiares no emparentados, que proceden de diferentes zonas del mismo país de origen. Se activan en destino para proporcionar ayuda a sus miembros durante un tiempo hasta que se produce un asentamiento más o menos definitivo, entonces se desactivan aunque se alimenta la relación en tanto que colectivo nacional.

"Yo tengo en Prades una prima de mi marido que llevaba aquí cuatro años. Ella estaba buscando trabajo en Internet y quiere trabajar y por eso vino aquí, al English Summer. (...) después su esposo, con niño, después otra familia y por ella nosotros aquí (...) Aquí en Prades hay diez familiares de $m$ marido y del resto de rumanos no son todos de la región de donde vengo yo, son de Moldavia, de Mar Menor, yo no los conocía de antes... Nos conocemos casi todos (...) no son todos de la misma religión, mi familia sí, pero los que son de otras partes de Rumanía, no, pero es igual hablamos con todos (...) el otro día hicimos todos una comida" (Adriana, Romania, Prades).

Sn interesantes los ejemplos de cadenas y redes de proyectos aparentemente familiares que, una vez en destino, de convierten en netamente individuales (Suárez y Crespo, 2007), por el arraigo en el territorio, aunque sin olvidar la presencia del resto del colectivo que hace funciones de red de ayuda e información pero con el que se mantiene una cierta equidistancia para evitar el control social que ejerce el colectivo nacional.

"Vine por un ex-novio que nos conocimos por foto a través de una amiga que venía y ella tenía dieciséis años y se casaba y venía a vivir aquí. Entonces empezamos a hablar por teléfono y nos gustamos solo por hablar por teléfono... y quería que viniera a vivir aquí, porque él ya vivía. Vine gustamos solo por hablar por teléfono... y quería que viniera a vivir aquí, porque él ya vivía. Vine
para conocerlo y a ver (...) al final estuvimos un año juntos, me llegó una oferta de trabajo y entonces para conocerlo y a ver (...) al final estuvimos un año juntos, me llegó una oferta de trabajo y entonces me quedé y él se fue con otra brasileña (...) Después vino mi hermana con su marido que es de mi
pueblo también... el marido de mi hermana vino a través de la chica que vine yo. Esta chica que me pueblo también... el marido de mi hermana vino a través de la chica que vine yo. Esta chica que me
presentó a mi ex novio, su hermano está casado con una prima de ella. Entonces trajo a su prima (...) Mi hermano también estaba aquí y yo traje a su novia que tenía 16 años y nosotros que somos más familia nos apoyamos mutuamente (...) Yo estoy muy bien aquí y no te digo con los brasileños, son muy pocos los que tengo relación, ¿vale? Porque cada uno tiene su vida y tal. Pero por más que estemos reunidos busco mantenerme al margen para evitar a veces discusiones, peleas (...) si se quieren quedar en mi casa, se pueden quedar en mi casa, no me importa nos ayudamos con trabajo a través de uno y de otro" (Joana, Brasil, Llavorsí).

Las mujeres con proyectos aparentemente individuales (Suárez y Crespo 2007) son las jefas de hogar (Oso, 1998) en origen y en destino (desde la distancia) vienen solas para trabajar, maximizar el ahorro y enviar dinero al resto del grupo familiar, con un proyecto de retorno claramente definido en origen que puede derivar en una decisión de permanencia en destino si proceden a la reagrupación familiar. Ocupan sectores laborales frecuentemente relacionados con la atención a la dependencia y forman cadenas y sectores laborales frecuen
redes muy feminizadas.

Las cadenas son funcionales $y$, en los pueblos, esta funcionalidad toma un relieve especial porque se hace fácilmente visible. Actúan como redes de obtención de ayuda e información, aportan capital social y recursos a sus miembros, fomentan la cultura de la migración, sustentan lazos económicos, afectivos, socioculturales, políticos y participativos de carácter transnacional; impulsan nuevos proyectos y también amortiguan el impacto de la movilidad descendente inherente a la migración (Pedone 2005). No obstante, las cadenas también tienen efectos perversos, como frenar la movilidad social y laboral, dificultar el contacto con la sociedad receptora al crear pequeños grupos de iguales, y el control socia que ejercen los miembros de la cadena, especialmente entre las mujeres. 


\subsection{Más allá del municipio: el territorio}

La mayor parte de las mujeres entrevistadas hablan de una fidelidad continuada al municipio desde su llegada porque éste ha satisfecho desde el primer momento sus expectativas personales y laborales. La fidelidad a la comunidad, es aún más reivindicada cuando se ha producido la reagrupación familiar cuando se convive con un grupo de compatriotas:

"Teníamos a mi cuñada aquí, la hermana de mi marido. Nos llamó (en 2008) para trabajar en e campo aquí en Benissanet, los dos. Después él ha quedado en el campo hasta el año pasado y yo he encontrado algunas casas donde trabajar para limpiarlas. Bueno estamos bien aquí y no nos pensamos marchar porque allí nos estamos haciendo la casa en Rumanía. Con el dinero que ganamos nos hacemos la casa y ya veremos, de momento no estamos decididos a marcharnos. Tenemos un trabajo fijo los dos, no pensamos marchar de momento" (Bibiana, Rumanía, Benissanet).

En ocasiones la decisión de quedarse puede ser la consecuencia de haber iniciado una relación con un hombre local y se piensa en un proyecto de familia en el municipio:

"Y a través de él conozco a un montón de gente. He viajado con él. Es buenísimo chico y me ha ayudado muchísimo. Si no fuera por él, yo ya no estaría aquí. Porque cuando me vine, me vine por un año, ¿vale? Y mira, cuatro años" (Blanca, Paraguay, Organyà).

Los relatos sobre sus trayectorias migratorias hasta llegar al municipio en el que actualmente viven también son ilustrados con itinerarios complejos, aunque más fieles a un territorio, que a un municipio. Estamos de acuerdo con Pedreño y Riquelme (2007) cuando afirman que los inmigrantes no llegan de manera involuntaria a los pueblos si no que muchos dirigen conscientemente su trayectoria hacia estos entornos. Los proyectos migratorios de mujeres solas con familia en origen (Ios aparentemente individuales) son conducidos a estos entornos por las ventajas económicas que conllevan. El hecho de no tener casa propia, ni familia, las desliga del municipio aunque tengan como referencia el territorio en el que trabajan:

"Yo estoy donde tengo trabajo, me da igual si estoy en Llavorsí o estoy en Sort o donde estoy (...) no me importa. Si encuentro en Sort o si encuentro en Lleida. Donde tengo trabajo allá me quedo" (Crina, Rumanía, Llavorsí).

Sobre todo en lugares montañosos y mal comunicados, con economías basadas en el turismo, las trabajadoras inmigrantes se desplazan fácilmente a vivir a uno o a otro municipio en función de las temporadas laborales y condicionadas también por las dificultades en el acceso a la vivienda. En los pueblos acostumbra a haber poca oferta y muy cara, además, el clima muy frío (que requiere una fuerte pueblos acostumbra a haber poca oferta y muy cara, además, el clima

"Aquí se alquiler se paga 550, amueblado, sí es muy caro el alquiler y otros piden 450 con dos habitaciones. Entre lo que gana mi marido y gano yo vamos apretando, vamos ahorrando. Esta es la ventaja del pueblo, que como no se sale... Porque cuando yo voy a Lleida te gastas la mitad del ventaja del pueblo, que como no se sale... Porque cuando yo voy a Lleida te gastas la mitad del
sueldo por eso no vamos todos los meses (...) Venimos a ahorrar pero entre los críos y comprarte cosas, para ahorrar... pero yo tengo calefacción, (...) yo la verdad es que no me privo" (Pilar, Bolivia Les).

Una buena parte de las informantes entrevistadas residen en el mismo lugar donde trabajan: la casa de la persona que cuidan, el hotel o el hostal donde que limpian, cocinan o hacen de camareras, los apartamentos del propietario del supermercado, etc. Muchas no tienen vehículo, ni siquiera carnet de conducir lo cual restringe su movilidad aún más si tenemos en cuenta las limitaciones del servicio de transporte público en las áreas rurales. Aún así, el territorio más amplio, sigue siendo la referencia, para comprar, pasear divertirse y trabajar:

"Cuando tengo libre, que no trabajo, me voy a algún sitio lejos como Lérida, Balaguer. El día que trabajo no puedo irme a un sitio lejos. Me quedo sola aquí en el pueblo (...) Más o menos está mejor pero antes era muy difícil para moverte con bus. Solo había un coche de línea y ahora hay dos pero antes era muy difícil para moverte con bus. Solo había
líneas. Para ir a Lérida hay dos" (Fátima, Marruecos, Llavorsí).

"Me voy por la mañana a las 8 con el autocar de Guimerà a Tàrrega, en Tàrrega hay dos chicas que trabajan en Velltall en el restaurante. Las llamo y me recogen. Subo con ellas llego a Belltall y me vuelvo a Guimerá con el panadero que lleva el pan. Me ducho y me cambio y cojo el autobús que va 


\subsection{Construir capital social: ser imprescindibles y estar disponibles}

El tercer aspecto con el que defendemos la singularidad de la migración de las mujeres en los pueblos es la especificidad del capital social. Éste generalmente se asocia a la propia red de soporte que los migrantes disponen en destino: familia, amigos, conocidos y connacionales. En el ámbito rural la capacidad de los migrantes para enriquecer su capital social en la sociedad de llegada es mayor. La pequeñez del entorno local permite el contacto directo con el tejido social. Los migrantes son fácilmente conocidos y acceden también con mayor facilidad al conocimiento de las redes locales que pueden facilitarles recursos esenciales como el trabajo y la vivienda.

"Al principio cuando comencé a trabajar aquí fui en busca de mi tarjeta médica y me dijo el enfermero ¿no tienes trabajo? Y yo le dije que no y me mandó donde una señora que se llama Carme que ahora está en la residencia. Y estuve con ella tres meses haciéndole cuatro horas. La gente donde yo tuve mi prime trabajo cuando llegué aquí, conocen mucho a las personas y como sabe cómo trabajo yo, pues le hablaron también al señor... de que yo podía cuidar (...) Aquí en Organyà yo tengo más facilidades, el pueblo es pequeño, conozco a la gene, la gente me conoce y yo puedo encontrar mejor un contrato de trabajo aquí que en Madrid (donde vive un nieto)" (Mari Paz, República Dominicana, Organyà).

El capital social se adquiere por la vía de la confianza que la población local deposita en la población inmigrada y, en segundo lugar, por la vía de la total disponibilidad laboral que expresan las mujeres. Hacerse con la confianza de la población local favorece la obtención de recursos de los migrantes y de los miembros de sus cadenas y redes:

"Uno es informático y trabaja de eso. Y otro trabaja en el bar del puente. Otro trabaja en el restauran de los Soya (...). Y mi hermano y dos de mis cuñados trabajan en la construcción y luego mis dos primas trabajan en el restaurant, $Y$ luego yo y mi hermana trabajamos en la carnicería. Y m concuñada, una es del supermercado. También es cajera acá. Otra está en lo del tabaco y otras dos

están ahora en el paro. Hay otra boliviana que cuida a la mamá de Sisco..." (Pilar, Bolivia, Les).

En los pueblos todo es mundo se conoce y las informaciones y referencias personales directas circulan rápidamente por las redes locales. Cuando alguien decide contratar a una persona extranjera indaga en las conversaciones informales con otros vecinos y vecinas y es aquí donde emerge otro de los pilares sobre los que se sostiene y alimenta el capital social: la disponibilidad.

"Hay gente, pero dependiendo que faena, no las coge nadie de aquí. Porque si es fin de semana, 'ah no, fin de semana no quiero trabajar, yo quiero el fin de semana libre'. Entonces a nosotros nos dicen, 'mira tenemos para el domingo, ¿me puedes venir a hacer un repaso?' Entonces yo digo: vale ¿cuántas horas? Por la noche, vale. Hasta ahora no hemos dicho no a nadie. En el restaurante aquí ¿cuántas horas? Por la noche, vale. Hasta ahora no hemos dicho no a nadie. En el restaurante aquí ¿n el Estanc, trabajé seis meses con él, me llama un domingo o un sábado, 'mira no viene mi hijo, ¿me ayudas?' Y yo no veo ningún problema, yo me voy (...). Yo no veo problema en trabajar por la
noche. También de panadero mi marido trabajó aquí, a ver, no había trabajo, pues de panadero. A la noche. También de panadero mi marido trabajó aquí, a ver, no había

Los trabajos que más abundan en el ámbito rural son duros y precarios: salarios bajos, a veces sin contrato, largas jornadas, horarios intempestivos e inestabilidad. La población local los rechaza y, en consecuencia, se convierten en una oportunidad para las personas inmigradas. Incluso quienes han conseguido una cierta estabilidad laboral y calidad de vida continúan mostrándose disponibles, algo que es bien valorado por los posibles empleadores y que las migrantes explotan como un valor.

\subsection{Incorporaciones fragmentadas}

$\mathrm{Ni}$ la disponibilidad, ni la confianza, ni las relaciones laborales, aseguran una completa y exitosa incorporación de las mujeres extranjeras al municipio. Aunque impera la presunción de que la inserción social de la población extranjera es más fácil y rápida en los pueblos que en las ciudades, comprobamos que persiste la división ente población local y extraniera incluso cuando "Ios foráneos" son conocidos. que per lise la divión enter Ser del pueblo implica haber nacido en la comunidad, tener raíces familiares en ella, y disponer de patrimonio o de capital simbólico. Las mujeres extranjeras, como cualquier otra población que, por motivos diversos (laborales, conyugales, familiares, o residenciales) ha llegado tradicionalmente a las pequeñas comunidades, están en el pueblo, incluso pertenecen a él, de una determinada forma, pero no son de la comunidad. En general, podemos afirmar que las comunidades locales se muestran reacias a la incorporación social de la población migrante, independientemente del modelo de desarrollo socio- 
En nuestra investigación pedimos a las informantes que nos detallaran particularidades negativas y positivas de vivir en un pueblo y comprobamos que en términos negativos, la mayoría sólo se refirió a aspectos puntuales (algunos ruidos, el clima). Quizás fueron las que venían de zonas más urbanas las que expresaron mayor insatisfacción. Y contrariadas también se mostraban aquellas que son conscientes de que su colectivo despierta desconfianza en la población local, la cual exhibe gestos de suspicacia evidentes, aunque, según ellas, justificables:

yo siempre saludo. Si me responden bien y si no me responden hay veces que me da lástima cuando me miran así. Hay muchos que no contestan y hacen una mirada muy... pero también hay gente buena y preguntas qué haces, cómo vives, si estás bien, si te gusta estar aquí (...). Eso es normal. Si yo estoy en mi pueblo, en mi país, cuando viene mucha gente de fuera también pensaría qué está pasando, van a coger todo, nos quedaremos sin nada. Como aquí (Zaida, Marruecos, Vila-rodona).

Las valoraciones positivas se centraron fundamentalmente en el entorno, la calidad de vida, las oportunidades laborales y de obtención de recursos económicos en general y en las relaciones personales, base de su prestigio y de la capacidad de conseguir capital social. En algunos casos, el municipio es vivido como si del espacio de trabajo se tratara, como un no lugar en el que se está, más o menos indefinidamente, de paso. Pero también hallamos itinerarios que progresivamente van siendo menos indefinidamente, de paso. Pero también hallamos itinerarios que progresivamente van siendo pensados como irreversibles que son los que afectan a las cadenas familiares formadas a partir de
sucesivos procesos de reagrupación. La decisión de permanecer tampoco garantiza la incorporación total a la sociedad local.

"Yo no formo parte de nada, no tengo tiempo (...). Con todos, hola, buenos día, buenas tardes y adiós. Voy con los niños a la plaza y me llevo bien con toda la gente. Pero la mayor parte de la gente te dicen hola, buenos días y adiós. Ya está" (Bernarda, Portugal, Vila-rodona).

Quienes han aplazado el retorno y quienes ni lo contemplaban desde el principio arraigan en el municipio a través de la compra de un piso, un empleo más o menos estable o la formación de una familia con un hombre local pero sin de dejar de sentir que a pesar de estar, no pertenecen a la comunidad:

"Hasta ahora me ha ido bien y no veo la idea de irme de Prades, tengo mi piso, tengo mi coche. Y de irme. El niño está contento de estar aquí (...). Esta es su casa '¿de dónde eres?' de Prades, dice. 'pero tú no naciste aquí' y entonces dice 'yo soy colombiano, pero soy de Prades'. Se ha hecho de aquí. ¿Será de aquí? Si lo aceptan, sí" (Berta, Colombia, Prades).

Las mujeres inmigradas no tienen oportunidad de cultivar las relaciones sociales más allá de lo estrictamente laboral. En algunos casos, la comunidad de origen ha comenzado a participar tímidamente en las fiestas locales. Pero la incorporación es difícil. La inserción laboral y su prestigio como trabajadoras, no va acompañada de la inserción en los tejidos sociales de los pueblos. En la calle se observa la segmentación de la sociedad local y las dificultades para incorporar nuevos vecinos y vecinas que aunque con su trabajo resuelven algunas de las dificultades que tienen las comunidades, sigue siendo percibidos como forasteros demasiado diferentes $y$, en gran medida, ajenos a la comunidad local.

\section{Atender la dependencia: las cuidadoras inmigrantes}

La nueva ruralidad adopta estrategias adaptativas para la atención a las persones mayores como resultado de la "crisis del cuidado" que afecta a nivel global, pero también como respuesta a los cambios sociales y demográficos que singularizan estos medios rurales: a) sobreenvejecimiento de la población y de las posibles cuidadoras; b) imposibilidad de recurrir a los relevos generacionales; c) la insuficiencia y mayor dificultad de acceso a servicios especializados de atención diurna (soporte necesario para que la persona pueda permanecer en su domicilio habitual); d) más dificultades, por lo tanto, para poder "envejecer en casa y en el pueblo", sentimiento fuertemente enraizado en el medio rural; e) gran dispersión del hábitat, lo que implica una mayor demanda de movilidad per acceder a los centros asistenciales que, generalmente, depende del transporte privado de los familiares o vecinos; $f$ ) disminución y, en algunos casos, inexistencia de la "generación de suporte", es decir, de personas con edad y nivel de autonomía adecuado para atender las situaciones de dependencia.

En las nuevas realidades rurales la presencia de mujeres inmigrantes permite la expansión y la 

extraído del contexto familiar-doméstico pero se puede mantener la consigna tradicional local, de "envejecer en casa". La familia se ve forzada a mercantilizar la actividad de cuidar a sus personas mayores, pero lo hace dentro del mismo espacio doméstico a través de la contratación de mujeres migrantes.

\subsection{La dimensión de género y de clase en la contratación de mujeres inmigrantes en el entorno} rural para atender la dependencia

La contratación de mujeres inmigrantes en la nueva ruralidad para atender a las personas mayores permite reorganizar el trabajo reproductivo a través de acciones individuales y con resultados inmediatos dentro de las coordenadas sociales, económicas y de género existentes en el medio rural. La inexistencia de diferentes generaciones de mujeres de una misma familia en el municipio y la inexistencia de mujeres locales con disposición para realizar estos trabajos, refuerzan la necesidad de buscar mujeres de fuera para atender unas necesidades concretas (las derivadas de la situación de dependencia), pero también para preservar así cuestiones sociales muy singulares del medio rural: el hecho de envejecer (también morir) en casa, la importancia de la posición social de "la casa" y la necesidad de preservar la privacidad frente el control social propio de estas comunidades. Existe presión social hacía los familiares responsables de las personas mayores y se constata que a mayor posición social más exigencia en el responsables de las personas mayores y se constata que a mayor posición social más exigencia en e
nivel de atención, que generalmente se expresa en la tipología de soportes que se adoptan. En este sentido la contratación de una mujer en la modalidad de interna es una cuestión de status (Pérez Orozco 2008, Agrela, Martín y Langa, 2010) y es percibida por la comunidad como una opción de prestigio y coherente con la posición social de "la casa".

\subsection{Una relación laboral informal y precaria}

Las mujeres inmigrantes, como sustitutas de las mujeres de la familias y del pueblo, en los trabajos de cuidado, dan un pleno sentido al concepto de "fuga del cuidado" (Bettio, Simonazzi, Solinas y Villa 2004) para describir un modelo de atención y cuidado basado en la fuerza de trabajo barata y flexible y una relación laboral caracterizada por la informalidad, la arbitrariedad, la asimetría y la precariedad en las condiciones de trabajo (Castelló 2008). Se trata de unas tareas que se inscriben en el ámbito relacional y de las emociones (Martín Palomo 2008) y que se caracteriza por la invisibilidad y discreción, atributos que coinciden con la situación de las mujeres inmigrantes, muchas de ellas en situación de irregularidad. que coinciden con la situación de las mujeres inmigrantes, muchas de ellas en situación de irregularidad. cuidado implican que sea un colectivo vulnerable el que se incorpore a esta actividad y, parece, que las cuidado implican que sea un colectivo vulnerable el que se incorpore a esta actividad y, parece, que las
mujeres inmigrantes encuentran en estos trabajos y en la invisibilidad del medio rural donde se mujeres inmigrantes encuentran en estos trabajos y en la invisibilidad del medio rural donde se
desarrollan, un doble contexto de oportunidad para iniciar su proyecto migratorio, que les permite vivir en desarrollan, un doble contexto de oportunidad para iniciar su proyecto migratorio, que les permite vivir en
situación irregular mientras consiguen regularizar los papeles. Por otro lado, las mujeres inmigrantes son reclamadas por su experiencia vital como mujeres y por su capacidad para desarrollar unas funciones que se estima han aprendido de "forma natural" como mujeres en su propia familia. Pero, su realidad es un poco más compleja, ya que desconocen el contexto, los patrones culturales de salud, de alimentación los sistemas de atención sanitarios y de servicios sociales. No siempre tienen capacidad de reacción sobre la trabajadora inmigrante una responsabilidad que deviene una carga de gran intensidad emocional que no es reconocida por los
empleadores.

La flexibilidad y disponibilidad de las mujeres inmigrantes son dos atributos que no tienen las mujeres locales y que los empleadores consideran necesarias para dar respuesta a la atención que requieren las personas mayores. Observamos tres factores que inciden en la contratación de la modalidad del servicio (externa o interna): el grado de dependencia, la proximidad residencial de los familiares y la posición socioeconómica de "la casa". Cuanto mayor es el nivel de dependencia más horas de presencia de la cuidadora contratada en el domicilio y, por lo tanto, más incidencia de la modalidad de interna independientemente de la proximidad de los familiares responsables.

La continuidad y la estabilidad de la relación laboral es un aspecto valorado por las dos partes de contrato. Para la mujer inmigrante significa asegurar los ingresos económicos durante todo el año, si bien se trata de una ocupación de carácter temporal dado que la relación laboral depende del tiempo de vida de la persona atendida. El familiar que contrata valora la estabilidad de la relación laboral en función del nivel de satisfacción que manifiesta la persona cuidada y del establecimiento de una relación de confianza con la cuidadora. 


\section{Conclusiones}

Los municipios rurales de Cataluña están asistiendo a un proceso de repoblación gracias a la llegada de población inmigrada que, procedente de los más diversos países, contribuye a desarrollar y sostener las nuevas formas de ruralidad que se han ido vertebrando a raíz del proceso de desagrarización. Las medidas de desarrollo rural, promovidas por las administraciones, han situado a los municipios como proveedores de servicios vinculados al turismo y a la explotación del patrimonio natural y cultural que atrae a hombres y mujeres, especialmente a las últimas, el trabajo de las cuales deviene imprescindible para la reproducción de las comunidades. La incorporación de las mujeres inmigradas a las zonas rurales reviste algunas singularidades.

En primer lugar, en la narrativa sobre la llegada al pueblo, las mujeres han reconstruido su cadena migratoria, una circunstancia que no es nueva en la migración pero que aquí toma más sentido porque se hace más evidente la existencia de estrategias de reagrupación familiar que sitúan en los escenarios locales a los miembros de las familias extensas de las migrantes. Resulta fácil identificar la mujer que encabezó la migración y reconstruir el funcionamiento de la cadena.

En segundo lugar, son trayectorias que se dirigen hacia un municipio no siempre pensado ni vivido como un asentamiento definitivo sino como un eje que articula un circuito de movilidad laboral acotado al un asentamiento definitivo sino como un eje que articula un circuito de movilidad laboral acotado án sobre recursos laborales; dichas redes desbordan los municipios conectando pueblos vecinos en un área sobre recursos laborales; dichas redes desbordan los municipios conectando pueblos vecinos en un área territorial por la que se mueven las mujeres inmigradas en busca de trabajo. Hallamos pequeños desplazamientos residenciales entre municipios próximos, y
abandono de la zona rural que supuso el primer asentamiento.

Tercero, la explotación del capital social de las mujeres pasa por el establecimiento de relaciones más intensas con la población local y por el manejo de relaciones a corta distancia para mantener la conexión con el territorio y sus recursos. Ellas acceden con relativa rapidez a las redes locales de relación, que les permiten ser conocidas y conocer. Trabajar en el sector servicios (sobre todo servicio doméstico y atención a la dependencia), les permite acumular cierto capital social en la comunidad cuando las familias contratantes quedan satisfechas con el trabajo realizado.

Cuarto, a pesar de ser conocidas y conocer el entorno social de los municipios, las mujeres tienen dificultades para incorporarse a la sociedad local. Ésta las valora por su aportación laboral, pero la integración productiva no facilita, necesariamente, su participación activa en otros aspectos de la vida de la comunidad.

Por último, la contratación de mujeres inmigrantes para atender personas mayores en los municipios, supone una estrategia de mercantilización y una respuesta adaptativa a la crisis global de las estructuras supone una estrategia de mercantilización y una respuesta adaptativa a la crisis global de las estructuras
de atención y cuidado de las personas debido al envejecimiento de la población, a la incorporación de la de atención y cuidado de las personas debido al envejecimiento de la población, a la incorporación de la mujer en el mundo laboral y la insuficiencia de los sistemas de atención institucionalizados. Reorganiza el trabajo reproductivo transfiriéndolo desde el ámbito doméstico e informal al mercado. Las migrantes
acceden a un mercado de trabajo donde los empleadores las valoran por su disponibilidad y flexibilidad acceden a un mercado de trabajo donde los empleadores las valoran por su disponibilidad y flexibilidad de vínculos afectivos y de confianza.

Finalmente, a pesar de que la ruralidad aporta ciertas singularidades a los itinerarios de incorporación laboral de las mujeres inmigradas, las características socioeconómicas asociadas a los distintos territorios analizados, no suponen una mayor facilidad/dificultad para la integración de estas mujeres en la vida de esas comunidades rurales.

\section{Notas}

1. Esta comunicación presenta parte de los resultados de una investigación finalizada en 2011: La migración de mujeres extranjeras al medio rural catalán en el contexto de la transformación económica y social de las comunidades locales. El proyecto fue financiado por la AGAUR (ARAF1 00047), dirigido por Montserrat Soronellas y con la participación de Yolanda Bodoque, Gemma Casal y Ramona Torrens, del Departamento de Antropología, Filosofía y Trabajo Social de la Universitat Rovira y Virgili; y por Jordi Departamento de Antropología, Filosofía y Trabajo Social de la Universitat Rovira y Virgili; y por Jordi
Blay y Santiago Roquer, del Departamento de Geografía de la misma universidad. Contamos también 
con la colaboración de Carla Aguilar y Mercedes González, estudiantes del Máster en Migraciones y Mediación Social de la URV.

2. Siguiendo a Pedone (2006), entendemos por cadena la transferencia de información y apoyos materiales que familiares, amigos y paisanos ofrecen a los potenciales migrantes para decidir o eventualmente, concretar el viaje, gestionar documentación o empleo y conseguir vivienda.

3. Pueden ser familiares, de amistad, de paisanaje, pero también especializadas: de género, laborales, religiosas, sentimentales, etc., sin que ninguna de estas posibilidades excluya la presencia de las demás.

4. En este vínculo la inmigración aparece como un importante recurso para hacer frente a las carencias de un Estado de Bienestar que no dispone de las medidas asistenciales necesarias para atender a las personas mayores (Martínez Buján, 2005).

5. Los datos de población que citamos en el texto proceden del padrón de habitantes a 01/01/2010, excepto cuando se indica lo contrario (fuente: IDESCAT).

6. La tasa de crecimiento acumulado es de 1.84 para el total de Cataluña y del 1.7 para los municipios rurales (fuente: IDESCAT, elaboración propia).

7. En 2000, el índice de envejecimiento de la población residente de 147.3, mientras que en 2010 era de 118.1 (IDESCAT, elaboración propia).

8. El $24.3 \%$ de la población del municipio es extranjera (2010, Idescat).

9. 29 de los cuales son extranjeros.

10. Con la ayuda financiera de la PAC, a través de programas LEADER.

11. Freginals y Prat de Comte en la província de Tarragona, y Guimerà, en la província de Lleida.

12. "Por el sólo hecho de no ser del pueblo (se refiere a la mujer inmigrante contratada) también se siente más libertad. Porque si es del pueblo, entonces todos saben lo que haces o lo que no haces. En cambio uno de fuera, si le tienes que dar una bronca, se la das y no pasa nada. En cambio en un pueblo al día siguiente lo ves y siempre estas más atado" (Sebastià, empleador).

\section{Bibliografía}

Agrela, B. (M. T. Martín y D. Langa)

2010 "Modelos de provisión de cuidados: género, familias y migraciones. Nuevos retos y configuraciones para las políticas públicas", Alternativas: cuadernos de trabajo social 17: 9-17.

Agustín, L. M.

2003 "Olvidar la victimización. Los migrantes como protagonistas", Developement, 43 (3): 30-36.

Anderson, $B$.

2007 "A very private business: exploring the demand for migrant domestic workers", European Journal of Women's Studies 14 (3): 247-264.

Arellano, M.

2006 "Trabajadoras latinoamericanas en España: migraciones laborales y género", Cuadernos de Relaciones Laborales 24 (1): 151-179.

Artal, C. (A. Pascual y M. Solana)

2006 "Trajectòries migratòries de la població estrangera a Catalunya. Les poblacions marroquina,

equatoriana i pakistanesa", Observatori de la immigració a Catalunya, Migracat.

http://www20.gencat.cat/docs/dasc/01Departament/08Publicacions/Ambits\%20tematics//mmigracio/04publiencolaboracio/altrespublicacions/2006trajectories estrangers. pdf

Asis, M. M. (S. Huang y B. Shirlena)

2004 "When the Light of the Home is Abroad: Unskilled Female Migration and the Filipino Family"

Singapore Journal of Tropical Geography, 25 (2): 198-215. 
Bayona, J. (y F. Gil)

2010 "Migraciones de españoles y extranjeros en las áreas rurales catalanas (1996-2006): ¿Fin de la despoblación o nuevo modelo territorial?, Boletín de la Asociación de Geógrafos Españoles, 53: 219-237.

Benería, L.

2005 "Trabajo productivo/reproductivo, pobreza, y la globalización de la reproducción: consideraciones teóricas y prácticas", Mientras tanto, 100: 89-108.

Berger, A. (y P. Chevalier)

2001 "Nouvelles fonctions tertiaires et dynamiques démographiques en milieu rural, Espace, Population Sociétés, 1-2: 69-88.

Bettio, F. (y otros)

2004 "The 'care drain' in the Mediterranean: notes of the Italian Experience", 25th Conference of the International Working Party Labour Market Segmentation, Australia.

Bodoque, $\mathrm{Y}$.

2009 "Hombres sin mujeres. La búsqueda de la reproducción de la sociedad a través de la mirada de la ficción social", Gazeta de Antropología, 25 (2) art. 47.

http://www.gazeta-antropologia.es/?p=2014

Bonamour, J.

2001 "El mundo rural ante el nuevo siglo. Retos y desafíos", en F. García, El mundo rural en la era de la globalización: incertidumbres y potencialidades. Madrid, MAPA.

Bourdieu, $\mathrm{P}$

2004 El baile de los solteros. Barcelona, Anagrama.

Bryceson, D. y Vuorela, U. (eds.)

2001 The transnational family. New european frontiers and global networks. London, Berg

Camarero, L. (coord.)

2009 La población rural de España. De los desequilibrios a la sostenibilidad social. Barcelona: Obra Social "La Caixa". Colección Estudios Sociales, 27.

\section{Castelló, L.}

2008 "La mercantilización y mundialización del trabajo reproductivo. El caso español", en XI Jornadas de Economía Crítica. Bilbao.

Colectivo IOÉ

1996 Procesos de inserción y exclusión social de las mujeres migrantes no comunitarias. Instituto de la

Mujer.

http://www.colectivoioe.org/ficheros externos/Procesos $\% 20$ de $\% 20$ insercion $\% 20$ v $\% 20$ exclusion $\% 20$ social\%20de $\% 20$ las $\% 20$ mujeres $\% 20$ inmigrantes $\% 20$ no $\% 20$ comunitarias Procesosinsercion-exclusion-muj-ext.pdf

Collantes, F. (y otros)

2010. El impacto demográfico de la inmigración en la España rural despoblada. Real Instituto Elcano.

D'Aubeterre, M. E.

1995 "Tiempos de espera: emigración masculina, ciclo doméstico y situación de las mujeres en San Miguel Acuexcomac, Puebla", en S. González y V. Sallez (coords.), Relaciones de género y transformaciones agrarias. México, El Colegio de México.

Deigiuli, $F$.

2007 "A job with no boundaries: home eldercare work in Italy", European Journal of Women's Studies, 14 (3):193-207.

\section{Esparcia, J.}

2002 "La creciente importancia de la inmigración en las zonas rurales de la Comunidad Valenciana", Cuadernos de Geografía, 72: 289-306. 
Etxezarreta, M. (y L. Viladomiu)

1997 "El avance hacia la internacionalización. Crónica de una década de la agricultura española", en J.

González y B. Gómez, Agricultura y sociedad en la España contemporánea. Madrid, CIS

García, A. (y D. Sánchez)

2005 "La población rural en Cataluña: entre el declive y la revitalización", Cuadernos Geográficos, 36 (1) $387-407$.

García, B.

2003 Sociedad rural y desarrollo. Madrid, MAPA.

2006 "Inmigración extranjera y ruralidad", Circunstancia, 10

Giddens, A. y Hutton, W. (eds.)

2001 En el Límite: la vida en el capitalismo global. Madrid: Tusquets.

Gómez, J.

2001 "La 'nuevas' funciones socioeconómicas y medioambientales de los espacios rurales", en F. García El mundo rural en la era de la globalización: incertidumbres y potencialidades. Madrid, MAPA.

Gregorio, C.

1998 Migración femenina. Su impacto en las relaciones de género. Madrid, Narcea.

Gregorio, C. $<\mathrm{br} / \mathrm{p}>2007$ "Trabajando honestamente en casa de familia: entre la domesticidad y la hipersexualización", Revista de estudios feministas. 15 (3): 699-716.

Gualda, E. (y M. Ruiz)

2004 "Migración femenina de Europa del Este y mercado de trabajo agrícola en la provincia de Huelva, España", Migraciones Internacionales, 2 (4): 36-65.

Hochschild, A. R.

2001 "Las cadenas mundiales de afecto y asistencia y la plusvalía emocional", A. Giddens y W. Hutton (coords.), En el Límite: la vida en el capitalismo global. Madrid, Tusquets.

King, R.

2002 "Towards a New Map of European Migration", International Journal of Population Geography, 8: 89106.

Le Gall, J.

2008 "Le lien Familial et la solidarité à l'épreuve de la migration: le cas de musulmanes libanaises à Montreal". Original inédito.

Letablier, M.T.

2007 "El trabajo de 'cuidados' y su conceptualización en Europa", en C. Prieto, Trabajo, género y tiempo social. Madrid, Editorial Hacer y Editorial Complutense.

Luken, V. (M. Solana y A. Pascual)

2011 "La trayectoria migratoria internacional de la población inmigrada en España: la incidencia de las redes familiares y otras características sociodemográficas", Scripta Nova. Revista Electrónica de Geografía y Ciencias Sociales, (XV. 357).

Martín, E

2008 "El impacto de género en las migraciones de la globalización: mujeres, trabajos y relaciones interculturales", X Coloquio Internacional de Geogrítica. Universidad de Barcelona.

Martín Palomo, M.T.

2008 "Los cuidados y las mujeres en las familias", Política y Sociedad 45 (2): 29-47.

Martínez Buján, R.

2005 "El cuidado de ancianos: un vínculo entre la inmigración y el envejecimiento", Panorama Social, 2: $85-97$.

2010 "Servicio doméstico y trabajo de cuidados. Hacia la privatización del cuidado familiar", Alternativas: cuadernos de trabajo social, 17: 157-179. 
Martínez Virto, L

2010 "Gestión de los cuidados, desnacionalización y precariedad laboral: una perspectiva comparada", Alternativas: cuadernos de trabajo social, 17: 221-235.

Molina, M.

S/F El desarrollo rural: problemática y estrategias de futuro. Documento sin publicar.

Monreal, P. (y A. Del Valle)

2010 "Las personas mayores como actores en la comunidad rural: innovación y empowerment", Athenea Digital 17: 171-187.

Morén, R. (y M. Solana)

2006 "La immigració en àrees rurals i petites ciutats d'Espanya. Un estat de la qüestió" Documents d'Anàlisi Geogràfica, 47:141-155

Oso, L.

1998 La migración femenina de mujeres jefas de hogar. Madrid, Ministerio de Trabajo y Asuntos Sociales, Instituto de la Mujer.

2005 "Las jefas de hogar en un contexto migratorio: modelos y rupturas", en F. Checa (ed ), Mujeres en el camino. El fenómeno de la migración femenina en España. Barcelona, Icaria.

2010 "Movilidad laboral de las mujeres latinoamericanas en España y empresariado étnico", Familias, niños, niñas y jóvenes migrantes. Rompiendo estereotipos. Madrid, IEPALA.

Parella, S.

2003 Mujer, inmigrante y trabajadora: la triple discriminación. Barcelona, Anthropos.

2006 "La 'vulnerabilidad social' de las mujeres inmigrantes no comunitarias a partir del estudio de sus pautas de inserción laboral en España".

http://redsirevista.cebs-es.org

Parreñas, R. S.

2005 "Long distance intimacy: class, gender and intergeneracional relations between mothers and children in Filipino transnational families", Global Networks, 5 (4) 317-336

Pedone, $\mathrm{Cl}$.

2003 Estrategias migratorias y poder. 'Tú siempre jalás a los tuyos'. Quito, Abya Yala PMCD.

2005 "Diversificación de las cadenas migratorias ecuatorianas hacia el mercado de trabajo agrícola de Murcia, España", en A. Pedreno y M. Hernández (coords.), La condición inmigrante: exploraciones investigaciones desde la región de Murcia. Murcia, Universidad de Murcia.

Pedreño, A. (y P. J. Riquelme)

2007 "La condición inmigrante de los nuevos trabajadores rurales", Revista Española de Estudios Agrosociales y Pesqueros, 211:189-238.

Perez Orozco, A

2008 "Insostenibilidad del sistema global de cuidados y alternativas feministas", en Mujeres, sexo, poder, economía i ciudadania. XX Taller Forum de Política Feminista. Madrid: Forum de Política Feminista.

Piscitelli, A.

2004 "El tráfico del deseo: interseccionalidades no marco do turismo sexual no Nordeste do Brasil", Quaderns de l'Institut Català d'Antropologia, 4:1-16.

Portes, A. (L. Guarnizo y P. Landolt)

2003 "El estudio del transnacionalismo: peligros latentes y promesas de un campo de investigación emergente" en La globalización desde abajo: transnacionalismo inmigrante y desarrollo. México FLACSO.

Ramírez, E.

2002 "Se fue ¿a volver? Imaginarios, familias y redes sociales en la inmigración ecuatoriana a España (1997-2000)", Iconos 14:32-45

Reigada, A.

2007 "Trabajadoras inmigrantes en los campos freseros: hacia una segmentación sexual y étnica de trabajo y la vida social", V Congreso sobre las Migraciones en España. Valencia. 
Ribas, N.

1999 Las presencias de la inmigración femenina. Un recorrido por Filipinas, Gambia y Marruecos en Cataluña. Barcelona, Icaria.

Roca, J. (y otros)

2008 Amor importado, migrantes por amor: la constitución de parejas entre españoles y mujeres de América Latina y de Europa del Este en el marco de la transformación actual del sistema de género en España, Instituto de la Mujer.

http://www.inmujer.migualdad.es/mujer/mujeres/estud_inves/766.pdf

Rodríguez, G.

2007 "La protección social de la dependencia en España. Un modelo sui generis de desarrollo de los derechos sociales", Política y Sociedad, 44(2): 69-85

Rodríguez Cabrero, G.

2007 "La protección social de la dependencia en España. Un modelo sui generis de desarrollo de los derechos sociales", Política y Sociedad 44(2): 69-85

Roquer, S. (y J. Blay)

2008 "Del éxodo rural a la inmigración extranjera: el papel de la población extranjera en la recuperación demográfica de las zonas rurales españolas (1996-2006)", Scripta Nova. Revista Electrónica de Geografía y Ciencias Sociales, XII, 270.

Sanz, J.

2007 "Entre cumplir y hacer cosas". Significados sociales y culturales en torno al envío de remesas de la emigración ecuatoriana en España", en Actas del V Congreso sobre la Inmigración en España. Valencia.

Sassen, S.

2003 "Contrageografías de la globalización. La feminización de la supervivencia", en Contrageografías de la globalización. Género y ciudadanía en los circuitos transfronterizos, Madrid, Traficantes de Sueños.

Solé, C. (y otros)

2008 Las trayectorias sociales de las mujeres inmigrantes no comunitarias en España. Factores explicativos de la diversificación de la movilidad laboral intrageneracional. Instituto de la Mujer.

http://www.inmujer.migualdad.es/mujer/mujeres/estud inves/798.pdf

Soriano, R. Ma

2006 "Voces de mujeres desde la inmigración: una comparativa entre el asentamiento de marroquíes en España y mexicanas en EE.UU.", The Center for Comparative Immigration Studies. San Diego: University of California.

http://www.ccis-ucsd.org/publications/wrkg133.pdf

Soronellas, M.

2006 Pagesos en un món de canvis. Família i associacions agràries. Tarragona, Publicacions URV.

Suárez, L.

2008 "La perspectiva transnacional en los estudios migratorios. Génesis, derroteros y surcos metodológicos", en J. García y J. Lacomba, La inmigración en la sociedad española, una radiografía multidisciplinar. Barcelona, Bellaterra.

Suárez, L. (y P. Crespo)

2007 "Familias en movimiento. El caso de las mujeres rumanas en España", Migraciones, 21: 235-257.

Stockdale, A.

2006 "Migration: Pre-requisite for rural economic regeneration?", Journal of Rural Studies, 22:354-366.

Trepat, E. (y A. Vilaseca)

2008 Ruralitat i Immigració. La integració de les persones immigrades en el món rural català. Barcelona Fundació Món Rural.

\section{UNFPA}

2006 Hacia la esperanza: las mujeres y la migración internacional. Informe sobre el estado de la población mundial.

http://www.unfpa.org/swp/2006/pdf/sp sowp06.pdf 
Zlotnik, H.

2003 "The global dimension of female migration"

http://www.migrationinformation.org/feature/display.cfm?ID=109 